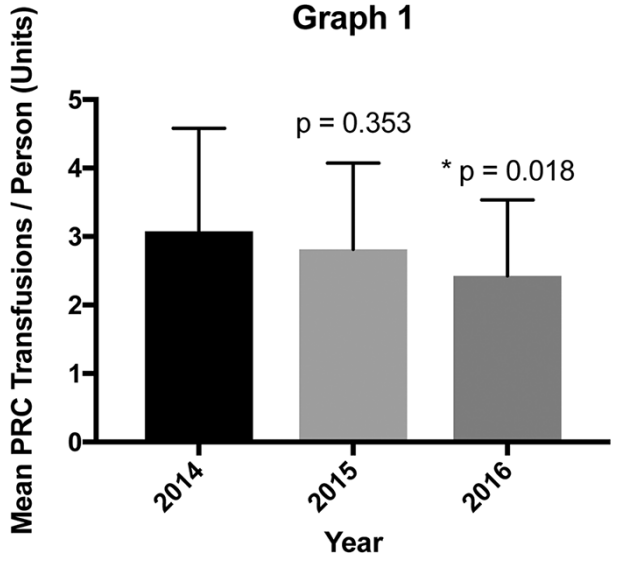

Abstract PTU-115 Figure 1
2014

2015

2016
Those who did not receive PRC transfusions or were transfused as outpatients or a semi-elective day case setting were excluded.

Results 321 of 770 episodes received PRC transfusion. The overall units transfused were: 468 in 2014, 334 in 2015 and 309 in 2016; equating to an equivalent of $93,600 \mathrm{mg}$, $66,800 \mathrm{mg}$ and $61,800 \mathrm{mg}$ of elemental iron (200 mg/unit). For those coded with anaemia $(n=137)$, there was a significant reduction in the mean PRCs transfused in 2016 compared to 2014 (figure 1).

With regards to IV iron preparations see table below.

\begin{tabular}{|c|c|c|c|c|c|}
\hline Year & $\begin{array}{l}\text { Total } \\
\text { Elemental } \\
\text { Iron }(\mathrm{mg})\end{array}$ & $\begin{array}{l}\text { Mean Dose/ } \\
\text { Administration } \\
\text { (mg) }\end{array}$ & $\begin{array}{l}\text { Ferinject } \\
500 \mathrm{mg} \\
\text { Doses }\end{array}$ & $\begin{array}{l}\text { Ferinject } \\
1000 \mathrm{mg} \\
\text { Doses }\end{array}$ & $\begin{array}{l}\text { Cosmofer } 100- \\
1600 \mathrm{mg} \\
\text { (Variable Doses) }\end{array}$ \\
\hline 2014 & 168400 & 923 & 10 & 87 & 86 \\
\hline 2015 & 229000 & 970 & 14 & 222 & 0 \\
\hline 2016 & 387500 & 969 & 25 & 375 & 0 \\
\hline
\end{tabular}

Using the same sample population as Graph 1, a significant increase in the administration of IV iron preparations is evident in 2016 compared to 2014 (Graph 2).

Conclusion Over three consecutive years, this retrospective study demonstrates an overall reduction in the number of PRC transfusions given to inpatients with anaemia, GIB, or menorrhagia, and an increase in IV iron $(\mathrm{mg})$ administered. Furthermore, for patients coded with anaemia in 2014 and 2016 , the data indicates a significant change in administration of both PRCs and IV iron. This suggests that the increased utilisation of IV iron contributes to a reduction in PRC transfusions, thereby lowering the potential risks associated with delivering blood products. This is particularly evident in those patients with anaemia as opposed to bleeding. Other reasons for the decrease in PRC transfusions may include the promulgation of a lower haemoglobin level at which to transfuse and the increased availability of elective day-case management of patients requiring transfusion.
Graph 2

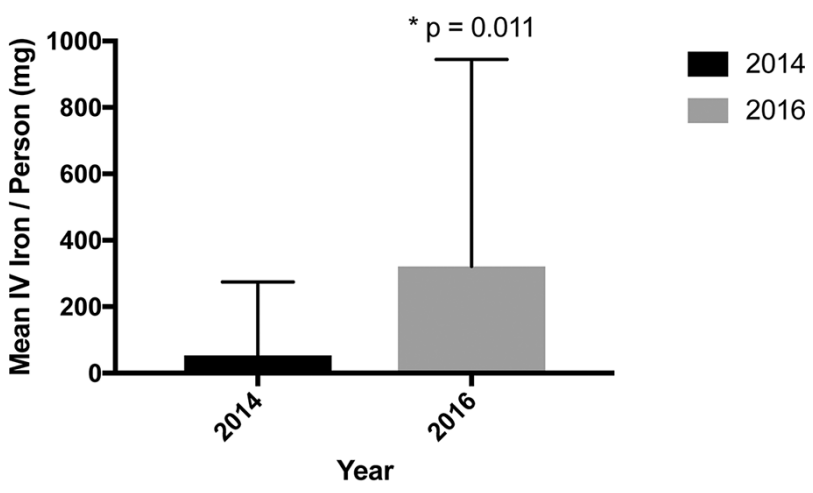

\section{PTU-116 INCREASED DIAGNOSIS AND TREATMENT OF HCV IN PRISON BY UNIVERSAL TESTING AND USE OF TELEMEDICINE}

1,2,35tuart McPherson*, ${ }^{3}$ Abi Hamoodi, ${ }^{3}$ Manoj Valappil, ${ }^{3}$ Dee Jones, ${ }^{3}$ Craig Thompson, ${ }^{3} J u l i e$ Dhuny, ${ }^{1} E m m a$ Robinson, ${ }^{1}$ Carolyn Miller, ${ }^{1}$ Margaret Hewett, ${ }^{1} E w a n$ Hunter, 1,3,4Sarah Morey. 'Viral Hepatitis Service, Newcastle Hospitals NHS FT, Newcastle Upon Tyne, UK ${ }^{2}$ Institute of Cellular Medicine, Newcastle University ${ }^{3}$ North East England Prison HCV Development Group ${ }^{4}$ Northumbria University

\subsection{6/gutjnl-2018-BSGAbstracts.494}

Introduction Chronic hepatitis $\mathrm{C}$ virus infection (HCV) is a major cause of end stage liver disease. With advances in antiviral therapy there is an opportunity to 'eliminate' HCV from the UK. HCV is common in incarcerated individuals $(\sim 7 \%$ of $\mathrm{UK}$ inmates are anti-HCV Ab positive). Increasing diagnosis and treatment of $\mathrm{HCV}$ in prison is a priority in order to achieve 'elimination'. However, HCV testing rates in UK prisons are low (4\%). In order to increase diagnosis and treatment of HCV in the North East of England (NEE) prisons we implemented: 1. A universal offer of blood borne virus testing (UBBVT) using dry blood spot testing for prisoners at reception to increase diagnosis; 2. Prison Telemedicine clinics within NEE Prisons to increase HCV treatment rates.

Aim To review results of the implementation of UBBVT in Durham Prison (DP) and the impact of the introduction of Telemedicine HCV treatment clinics in Northumberland Prison (NP).

Results UBBVT was implemented at DP, the major remand prison in NEE, in Mar 2016. From Mar 2016 to Feb 20173309 BBVT offers were made in total with 2831 of the 4280 (66\%) new receptions (NRs) were offered BBV testing, compared with 164 of 7,000 NRs (2.3\%) in 2013-14. 1495 (53\% of offered; 35\% of all NRs) NRs accepted BBV testing, of whom 95 (6.4\%) were anti HCV antibody positive. Of these $47(49.5 \%)$ were HCV RNA positive confirming active infection (3.1\% of all tested). Common reasons for non-acceptance of the test were 'doesn't want it' (54\%) and 'already had test' (37\%). Consultant-led Telemedicine clinic (TC) with nurse in-reach was implemented in NP (a medium sentence prison) in Aug 2015. Between Aug 2015 \&amp Oct 2017 80 individuals were seen in the TC. Of those seen in the TC, 57 (71\%) commenced anti-HCV treatment. In 1 year prior to implementation of the TC, only 6 patients received HCV treatment. 
Overall, satisfaction with the TC among the prisoners was very high ( $80 \%$ good or excellent). Moreover, this is very cost effective with reduced cost of prisoner movement (Est $£ 500 /$ hospital visit). SVR and adherence data will be presented.

Conclusions A universal offer of BBV testing to prisoners at reception to prison can substantially increase testing rates and lead to many new diagnoses of HCV. Prison telemedicine clinics with nurse-led in-reach offer a cost effective and efficient method of treating $\mathrm{HCV}$ in the prison environment.

\section{PTU-117 THE IMPACT OF AN ACUTE JAUNDICE CLINIC AT A TERTIARY REFERRAL CENTRE}

James Morgan*, Denise Gocher, Esther Unitt. University Hospital Coventry And Warwickshire, Coventry, UK

\subsection{6/gutjnl-2018-BSGAbstracts.495}

Introduction Jaundice is not a particularly common presentation in general practice (56 in 100,000). However it often indicates a serious underlying condition (35\% malignancy) which requires urgent investigation via a 2 week wait referral. ${ }^{1}$ Various methods have been tried to expedite these referrals including a rapid access hotline. ${ }^{2}$ and clinics. The experience and impact of an acute jaundice clinic providing prompt clinical, biochemical and radiological assessment is evaluated at a tertiary referral centre.

Methods The acute jaundice clinic provides open access biweekly clinics, following primary care referral, for clinical assessment, same day access to radiological investigations and prompt referral for hepato-pancreato-biliary (HPB) MDT discussion and, if required, biliary decompression. The primary goal is to ensure patients found to have HPB cancers are assessed quickly; the secondary aim is to avoid unnecessary admissions. This review will analyse appropriateness of referral, timing of investigations, diagnoses made and subsequent patient outcomes. Data was collected contemporaneously and supplemented with online patient records. This included patient age, bilirubin level, referral date and date of clinic appointment, timeliness of radiological investigations, final diagnosis, date of discussion at HPB MDT and malignant patient outcomes.

Results Data analysis was completed for all patients seen in the jaundice clinic over a 3 year period (2015-2017). In total, 291 patients were referred with a median age of 68 years (range 18-96 years). 245 (84.2\%) of these were deemed appropriate to be seen with $172(70 \%)$ clinically jaundiced at the time of review. Median time from GP referral to jaundice clinic review was 5 days (range 1-33 days). 209 (85.3\%) of the patients were managed in the outpatient setting. The main diagnoses made following jaundice clinic are shown in figure 1 .

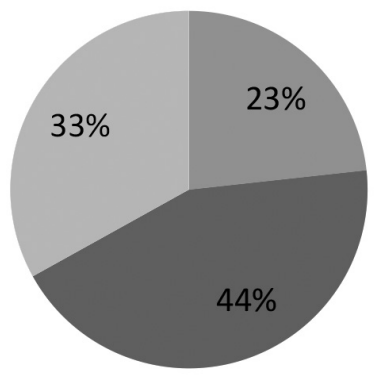

\section{Malignant \\ Gall stone related}

Other

Abstract PTU-117 Figure 1 Diagnoses made from jaundice clinic (percentage of patients)
For suspected malignant diagnoses, $90.4 \%$ had a CT on the day of clinic and were discussed at MDT, on average, 10 days (range 1-50 days) later. Outcome data was only available for 2016 and 2017 but in patients diagnosed with malignant biliary obstruction, 17/19 (89.5\%) had prompt biliary decompression with one of the remaining patients declining intervention. Only 4/19 (17.3\%) were eligible for potentially curative surgery and $5 / 19(26.3 \%)$ received palliative chemotherapy. One year survival was 50\% (9/18).

Conclusions The introduction of a jaundice clinic in a tertiary centre has been successful in providing timely review of jaundiced patients with high patient satisfaction. It has also allowed for prompt radiological assessment of potential malignant cases within 24 hours in more than $90 \%$ of cases with patients on average discussed in the HPB MDT within 10 days of jaundice clinic. The service has also proved extremely beneficial in avoiding admission in over $85 \%$ of patients. The remaining number admitted denotes the acute requirement for biliary decompression in a group of patients who often have significant co-morbidities. Disappointingly curative resection rates remain low in this cohort of patients, although this likely reflects the late stage of disease when jaundice is present and highlights the need for research into other predictors.

\section{REFERENCES}

1. Whitehead, MW, Hainsworth, I, Kingham, J. G. C. (2001). The causes of obvious jaundice in South West Wales: perceptions versus reality. Gut, 48(3),409-413.

2. Mitchell, J., Hussaini, H., McGovern, D., Farrow, R., Maskell, G., Dalton, H. (2002). Quality improvement report: The 'jaundice hotline' for the rapid assessment of patients with jaundice. BMJ: British Medical Journal, 325(7357),213.

\section{PTU-118 PRESENTING PROBLEMS AND OUTCOMES OF WOMEN WITH GYNAECOLOGICAL CANCER ATTENDING A SPECIALIST GI SYMPTOM CLINIC}

${ }^{1}$ Ann Muls*, ${ }^{1}$ Susan Lalondrelle, ${ }^{1}$ Alexandra Taylor, ${ }^{2}$ Christine Norton, ${ }^{3}$ Ailsa Hart, ${ }^{4}$ Jervoise Andreyev. ${ }^{1}$ The Royal Marsden NHS Foundation Trust, London, UK ${ }^{2}$ King's College London, London, UK ${ }^{3}$ Imperial College London, London, UK ${ }^{4}$ United Lincolnshire Hospitals NHS Trust, Lincoln, UK

\subsection{6/gutjnl-2018-BSGAbstracts.496}

Introduction Worldwide $1,470,900$ women are diagnosed yearly with a gynaecological cancer. Some women develop long-term changes in bowel function following treatment severely impacting on quality of life.

Methods A service evaluation collecting prospective data was approved by the organisation's $\mathrm{R}$ and $\mathrm{D}$ department. Intestinal symptoms were measured using a modified GSRS and impact on QoL assessed by VAS score $(0=$ worst QoL, $10=$ best QoL). The McNemar Chi-square and Wilcoxon signed rank tests were used to analyse changes in symptom burden between initial assessment to discharge from the service.

Results From April 2013 to March 2016, 235 women treated for gynaecological cancers attended the clinic, representing a fifth of those using the service. Fifteen declined further intervention and were excluded, leaving 220 for analysis. Women had been treated for cancer of the cervix (50\%), endometrium $(28 \%)$, ovary $(15 \%)$, vagina or vulva $(7 \%)$ cancer. Most women received multi-modal therapies: chemoradiation $(28 \%)$, surgery + radiotherapy (27\%), surgery +chemoradiation (22\%), surgery + chemotherapy (10\%). Median age was 57 years (range: 24-83). The median time between cancer diagnosis and referral to service was 4 years and 10 months 